\title{
Utilization of the MF Band for Providing Digital Radio Services in Greece
}

\author{
A. D. Papatsoris and D. Varsamis \\ Department of Engineering Informatics, Central Macedonia Institute of Technology, 62124 Serres, Greece.
}

Received 30 June 2015; Accepted 25 January 2016

\begin{abstract}
Many national and international broadcasters have recently realized the advantages of a complementary digital broadcast system at lower frequencies and especially below $30 \mathrm{MHz}$. Nevertheless, the inherent limited fidelity of existing AM services is causing listeners to search for other alternatives. In this paper, the propagation characteristics of the Medium Frequency band are explored for providing acceptable quality digital radio services of nationwide coverage in Greece.
\end{abstract}

Keywords: Digital Radio Mondiale, Radio Propagation, Ground Waves, OFDM, Single Frequency Network, Medium Frequency Broadcasting, International Telecommunications Union.

\section{Introduction}

Countries around the world are in the process of migrating from analogue to digital broadcasting techniques for both television and radio. In this paper the utilization of the Medium Frequency band for providing digital radio services of nationwide coverage in Greece is investigated and a typical SFN implementation is proposed.

Digital Radio Mondiale (DRM) [1] is a digital sound broadcasting system to replace existing analogue AM broadcasting in the bands below $30 \mathrm{MHz}$ (long-wave, medium-wave and short wave). The technology provides near-FM sound quality and also provides data content for display on a DRM receiver.

The major commercial advantage of digital broadcasting is the ability to offer higher quality and diversity of services. Since this can be done without the need for additional spectrum and with lower transmitter power this is attractive from the broadcaster's perspective. There are quite a few studies which have already dealt with both the technical and regulatory as well as commercial issues concerning the development of digital radio at frequencies below $30 \mathrm{MHz}$, and lately even at higher frequencies including HF and VHF portions of the spectrum. This has resulted into the introduction of relevant ITU-R Recommendations such as Recommendation ITU-R BS.1514 [2] describing a reference digital system suitable for broadcasting in the bands below $30 \mathrm{MHz}$.

Some key technical characteristics of the DRM system is that it provides for three different audio codecs of variable quality, application and bit rate requirements. Furthermore, COFDM with QAM linecode is used for the channel coding and modulation, along with time interleaving and forward error correction (FEC) using multi-level coding (MLC) based on a convolutional code. Pilot reference symbols are used to derive channel equalization information at the receiver. These features enable the realization of the DRM

\footnotetext{
*E-mail address: profadp@gmail.com
}

ISSN: $1791-2377$ @ 2016 Eastern Macedonia and Thrace Institute of Technology. All rights reserved. system within a single frequency network (SFN) architecture, similar in concept to those widely implemented in Digital Television.

Nevertheless, radio coverage of the DRM system is largely dictated by the propagation characteristics of radiowaves. In this paper we deal extensively with the groundwave mode of propagation, -which is applicable at medium frequencies (MF band)- in order to determine the radio coverage of a DRM single frequency digital radio network covering the entirety of Greece.

\section{Planning procedure for DRM30}

Planning tools for calculating coverage and availability of DRM transmissions are generally not yet available. Nevertheless, nominal systems have been analyzed and planning data for DRM networks have been derived from a mixture of theoretical and simulation modelling, complemented by laboratory and field trial measurements [3, 4]. The starting point for this work is a set of theoretical minimum carrier-to-noise ratios for the DRM system, which assume perfect receivers and no man-made noise or interference. It then becomes possible to predict the resultant radio coverage adopting the ITU planning assumptions and methodology, as typically suggested in Report ITU-R BS.2144 [5].

In the DRM specification, four robustness modes with different parameters (sub-carrier number and spacing, useful symbol and guard interval length, etc.) for the orthogonal frequency division multiplex (OFDM) transmission scheme are defined for the various propagation conditions in the LF, MF and HF bands (see Table 1).

Six predefined channel models are commonly specified as reference models for planning DRM radio coverage. The models are not relying just on theoretical analysis, but have been refined through a collection of experimental data based on real-world channel sounding experiments that were used to characterize Doppler, delay spread and multipath characteristics. These six channel models are defined in detail in ITU_R BS.1615-1 [6] and are shown in Table 2. 
Table 1. DRM robustness modes

\begin{tabular}{c|c|c}
\hline $\begin{array}{c}\text { Robust } \\
\text { ness } \\
\text { mode }\end{array}$ & Typical propagation conditions & $\begin{array}{c}\text { Preferred } \\
\text { frequency } \\
\text { bands }\end{array}$ \\
\hline A & Ground wave channels with minor & LF, MF \\
B & Time and frequency selective & MF, HF \\
C & As robustness mode B, but with & Only HF \\
D & As robustness mode B, but with & Only HF \\
\hline
\end{tabular}

Table 2. Summary of DRM30 RF channel models

\begin{tabular}{|c|c|c|c|c|}
\hline \multicolumn{2}{|r|}{ Channel model No. } & \multicolumn{3}{|c|}{ Representative of: } \\
\hline & & Good & Typical & Bad \\
\hline 1 & Additive white Gaussian noise & $\begin{array}{ll}\text { LF } & \text { MF } \\
\text { HF } & \end{array}$ & $\begin{array}{c}\text { LF with } \\
\text { variable } \\
\text { S/N }\end{array}$ & \\
\hline 2 & Ground-wave and sky-wave & & MF, HF & \\
\hline 3 & 2-path delay spread $2.2 \mathrm{~ms}$ & & $\mathrm{HF}$ & MF \\
\hline 4 & 2 equal paths delay spread $2 \mathrm{~ms}$ & & & $\mathrm{HF}$ \\
\hline 5 & 4 equal paths delay spread $4 \mathrm{~ms}$ & & & $\mathrm{HF}$ \\
\hline 6 & $\begin{array}{l}\text { Near vertical incidence in } \\
\text { tropical zones }\end{array}$ & & & $\begin{array}{c}\mathrm{HF} \\
\text { (NVIS) }\end{array}$ \\
\hline
\end{tabular}

Of particular interest to our study is channel model 1, which is applicable to MF broadcasting. Channel model 1 refers to a channel which is only subject to additive white Gaussian noise. Table 3, shows the required signal-to-noise ratio for all DRM30 robustness modes when operating in channel 1. A bit-error ratio of $1 \times 10^{-4}$ corresponds to the point at which subjective audio quality starts to degrade to the point that is defined as 'limit of service'. Similar tables for all six channels can be found in [2].

Table 3. S/N (dB) to achieve BER of $1 \times 10^{-4}$ for all DRM30 robustness modes for channel model 1.

\begin{tabular}{l|l|c|c|c|c|c}
\hline $\begin{array}{c}\text { Modulation } \\
\text { scheme }\end{array}$ & $\begin{array}{l}\text { Protect } \\
\text { ion } \\
\text { level } \\
\text { No }\end{array}$ & $\begin{array}{l}\text { Average } \\
\text { code rate }\end{array}$ & \multicolumn{2}{l}{$\begin{array}{l}\text { Robustness mode } \\
\text { occupancy type }\end{array}$} \\
\cline { 4 - 7 } & & $\mathrm{A} / 2$ & $\mathrm{~B} / 3$ & $\mathrm{C} / 3$ & $\mathrm{D} / 3$ \\
\cline { 4 - 7 } & & & $(9 \mathrm{kHz})$ & $\begin{array}{l}(10 \\
\mathrm{kHz})\end{array}$ & $\begin{array}{l}(10 \\
\mathrm{kHz})\end{array}$ & $\begin{array}{l}(10 \\
\mathrm{kHz})\end{array}$ \\
\hline 16 QAM & 0 & 0.5 & 8.6 & 9.3 & 9.6 & 10.2 \\
& 1 & 0.62 & 10.7 & 11.3 & 11.6 & 12.1 \\
\hline \multirow{2}{*}{64 QAM } & 0 & 0.5 & 14.1 & 14.7 & 15.1 & 15.9 \\
& 1 & 0.6 & 15.3 & 15.9 & 16.3 & 17.2 \\
& 2 & 0.71 & 17.1 & 17.7 & 18.1 & 19.1 \\
& 3 & 0.78 & 18.7 & 19.3 & 19.7 & 21.4 \\
\hline
\end{tabular}

\subsection{DRM minimum usable field strengths (MUFS)}

Substantial work has been carried out for determining the protection ratios and minimum usable field strength for the various DRM modes. Especially for DRM30, reference data have been published in [6].

As DRM is expected to work alongside the existing AM services for some considerable time, the planning process used is based on the same underlying principles and assumptions as those used for traditional AM services. For
AM planning purposes the MUFS is based on an audio S/N ratio of $26 \mathrm{~dB}$ with reference to $30 \%$ modulation and intrinsic receiver noise dependable on frequency and expressed as an equivalent noise electric field strength [7].

For digital systems a suitable BER for which perceived audio quality is considered good is chosen (in our case a value of BER of $1 \times 10^{-4}$ ). Subsequently, the receiver intrinsic noise at the frequency band of interest is calculated and the required carrier-to-noise ratio added to that for delivering to the audio receiver the chosen robustness mode service. For the MF band $(526.5 \mathrm{kHz}-1606.5 \mathrm{kHz})$ the receiver intrinsic equivalent noise electric field is $24.5 \mathrm{~dB} \mu \mathrm{V} / \mathrm{m}$ and thus the minimum usable field strength should be $24.5 \mathrm{~dB}+\mathrm{x} \mathrm{dB}$, where $\mathrm{x}$ is the required $\mathrm{S} / \mathrm{N}$ ratio to achieve BER of $1 \times 10^{-4}$ for all DRM30 robustness modes for channel model 1 . Values of $\mathrm{x}$ are given in Table 3.

Assuming a reception environment which corresponds to minimum noise conditions (such as quiet rural regions), the MUFS for various robustness modes can now be calculated. Table 4 illustrates minimum electric field strength values for planning purposes for robustness mode A.

Table 4. Minimum usable electric field strength $[\mathrm{dB} \mu \mathrm{V} / \mathrm{m}]$ to achieve BER of $1 \times 10^{-4}$ for DRM robustness mode A with different spectrum occupancy types dependent on protection level and modulation scheme for the MF frequency band (ground-wave propagation).

\begin{tabular}{c|c|c|c|c}
\hline \multirow{2}{*}{$\begin{array}{c}\text { Modulation } \\
\text { scheme }\end{array}$} & \multirow{2}{*}{$\begin{array}{c}\text { Protection } \\
\text { level No }\end{array}$} & \multirow{2}{*}{$\begin{array}{c}\text { Average } \\
\text { code rate }\end{array}$} & & \multicolumn{2}{|c}{ Robustness mode / spectrum occupancy type } \\
\cline { 4 - 5 } & & & $\mathrm{A} / 0(4.5 \mathrm{kHz})$ & $\mathrm{A} / 2(9 \mathrm{kHz})$ \\
\cline { 4 - 5 } & & & $\mathrm{A} / 1(5 \mathrm{kHz})$ & $\mathrm{A} / 3(10 \mathrm{kHz})$ \\
\hline \multirow{2}{*}{ 16 QAM } & 0 & 0.5 & 33.3 & 33.1 \\
& 1 & 0.62 & 35.4 & 35.2 \\
\hline \multirow{2}{*}{$64 \mathrm{QAM}$} & 0 & 0.5 & 38.8 & 38.6 \\
& 1 & 0.6 & 40.3 & 39.8 \\
& 2 & 0.71 & 42 & 41.6 \\
& 3 & 0.78 & 43.7 & 43.2 \\
\hline
\end{tabular}

\section{Radio propagation at the MF band}

Radio wave propagation at the lower part of the spectrum is described by several models. Apart from consideration of the near field which is needed when the wavelength is very large, the process involves three types of propagation: ground wave, sky wave and guided electromagnetic wave. The ground wave theory assumes that the propagation path is around a spherical earth with homogeneous parameters, and ignores the effect of the Ionosphere. Sky wave theory takes into consideration the effect of the Ionosphere where it is appropriate, mainly in the LF and MF bands, but at very low frequencies and low launch angles the similarity between the effective height of the Ionosphere and the wavelength of propagation makes the theory of guided waves in spherical waveguides more appropriate.

Ground wave propagation operates mainly in the three frequency bands, VLF, LF and MF, or $3 \mathrm{kHz}$ to $3 \mathrm{MHz}$, with some applications extending into the ELF and HF bands. The estimation of the electric field strength in the frequencies of interest, i.e. $(526.5 \mathrm{kHz}-1606.5 \mathrm{kHz})$ is based on the work of Rotheram [8] and Millington [9].

\subsection{Development of MF radio propagation tool}

For studying and specifying a broadcasting radio Single Frequency Network operating at the MF band, which is 
intended to offer nationwide coverage in Greece, a suitable radio propagation tool has been developed. The radio propagation tool provides estimation of the resultant electric field applicable to ground-wave propagation at the MF band.

The ground parameters of permittivity and conductivity for Greece [10] are shown in Fig. 1.

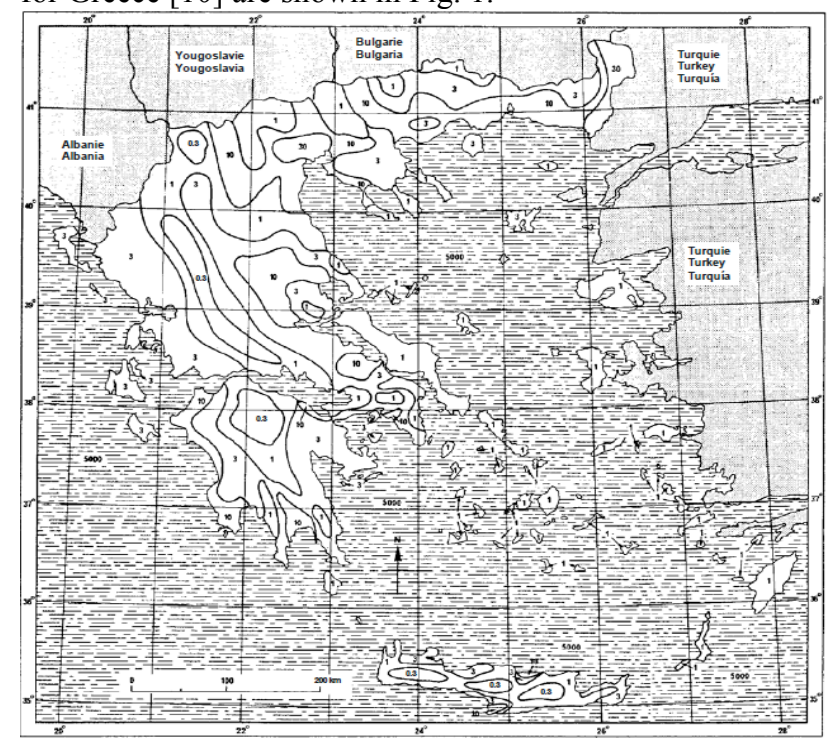

Fig. 1. MF map of ground conductivity for Greece.

The relevant conductivity $\sigma$ and relative permittivity $\varepsilon_{r}$ values have been adapted and superimposed onto the geographical map of Greece in order to develop appropriate Geotiff maps of conductivity, suitable for calculating the resultant electric field strength through different types of ground. Through a simple Graphical User Interface (GUI) the resultant maps were imported and facilitated in MATLAB's environment for ground wave propagation calculations. An arbitrary number of radio transmitters (Tx) and their corresponding technical parameters such as power, height, antenna type, etc. can be introduced. Subsequently, elaborate propagation calculations along radial paths can be performed at the desired angular accuracy to provide the resultant electric field strength.

\section{A Single Frequency Network implementation for providing nationwide digital radio broadcasting in Greece}

In order to demonstrate the utility of a single frequency radio broadcasting network in the MF band, a typical middle-band frequency of $1 \mathrm{MHz}$ has been chosen for our radio coverage calculations. Seven low-power transmitters of $1 \mathrm{~kW}$ have been placed in the locations shown in Table 5 .

Table 5. Characteristics of MF radio transmitters for the provision of nationwide coverage.

\begin{tabular}{c|c|c}
\hline Location & Power $[\mathbf{k W}]$ & Height $[\mathbf{m}]$ \\
\hline Apolonia (Sifnos) & 1 & 200 \\
Pirgos & 1 & 300 \\
Arta & 1 & 200 \\
Lamia & 1 & 500 \\
Ptolemaida & 1 & 500 \\
Serres & 1 & 200
\end{tabular}

Fillyra

1

200

The calculations have been performed for four sub-cases of robustness mode A/2 $(9 \mathrm{kHz})$, which is applicable to Europe according to the Geneva 1975 Assignment Plan for the LF and MF bands. Those sub-cases numbered from 1 to 4, correspond to a 16-QAM modulation scheme with protection levels 0 and 1 and a 64-QAM modulation scheme with protection levels 0 and 3, accordingly. Figures 2 to 4 show indicative radio coverage maps for the sub-cases 1 to 4 at Appolonia (Sifnos), Fillyra and Arta.

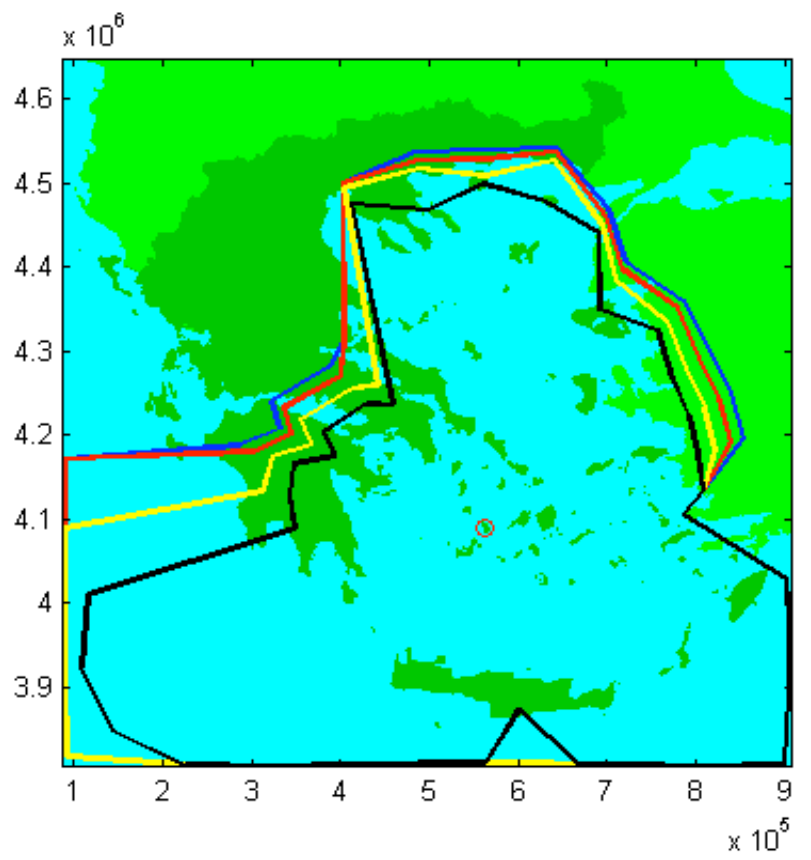

Fig. 2. Iso-electric field coverage curves of Appolonia (Sifnos) Tx.

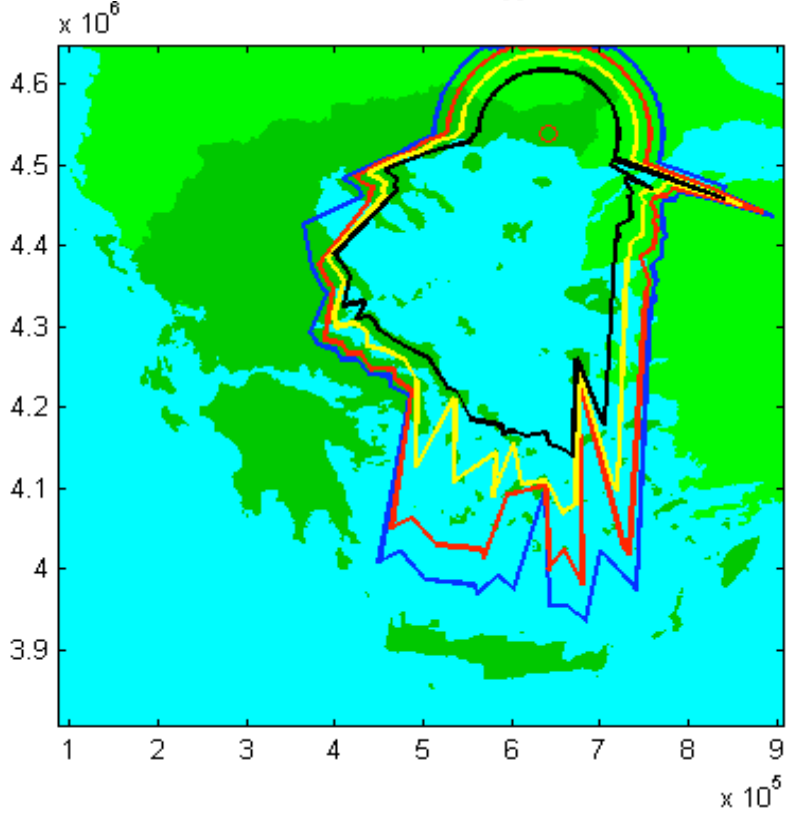

Fig. 3. Iso-electric field coverage curves of Fillyra Tx.

The blue, red, yellow and black lines correspond to robustness mode A/2 sub-cases 1 (16-QAM protection level 0), 2 (16-QAM protection level 1), 3 (64-QAM protection level 0 ) and 4 (64-QAM protection level 3), respectively. It is worth noting that the radio coverage area of sub-case 4 is significantly reduced when compared to sub-case 1 , as there is a difference of about $10 \mathrm{~dB} \mu \mathrm{V} / \mathrm{m}$ in terms of MUFS. 


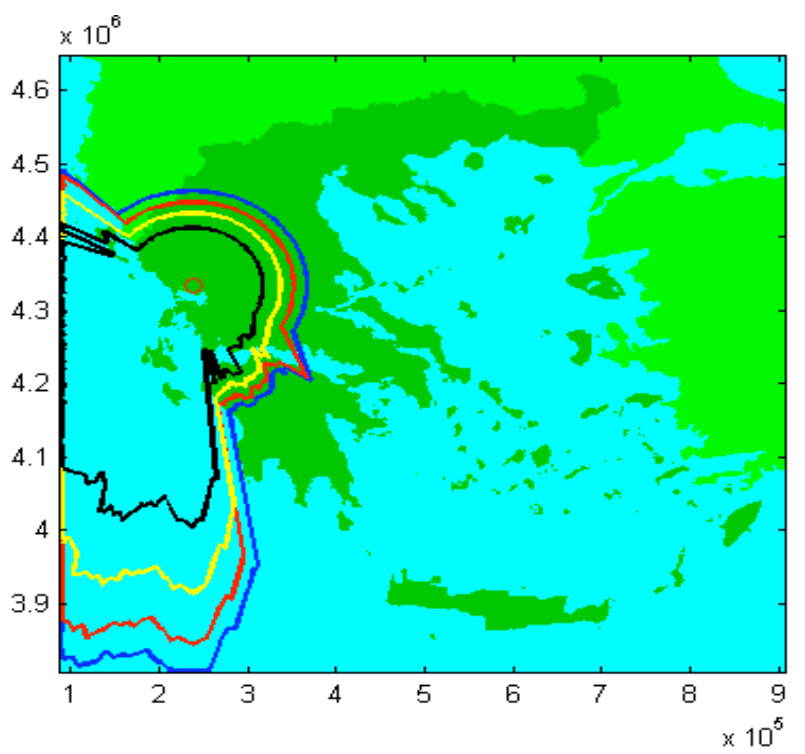

Fig. 4. Iso-electric field coverage curves of Arta Tx.

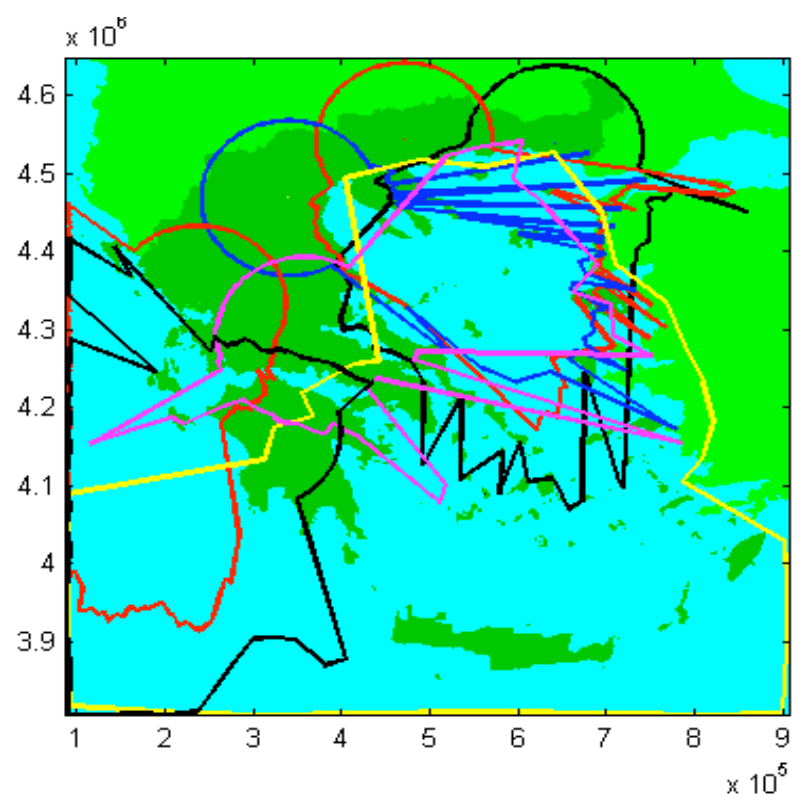

Fig. 5. SFN radio coverage for MF digital broadcasting Txs operating at $1 \mathrm{MHz}$ at robustness mode $\mathrm{A} / 2$, sub-case 3 .

Finally, Figure 5 shows a SFN implementation of a DRM30 network comprising of all seven low power transmitters operating at a frequency of $1 \mathrm{MHz}$ providing nationwide coverage in Greece broadcasting at robustness mode A/2 modulated with 64-QAM and protected at level 0 (Minimum usable field strength $38.6 \mathrm{~dB} \mu \mathrm{V} / \mathrm{m}$ ).

It can be clearly seen, that using a total transmitter power of $7 \mathrm{~kW}$ digital radio services of comparable sound quality to FM can be offered to the whole of Greece. By carefully placing the transmitters in the vicinity of densely populated areas, it is expected that radio coverage should not suffer from the increase of the noise floor, typically expected at urban areas. Nevertheless, for urban environments, the main factor affecting reception in the MF band is man-made noise. Depending on the density of urbanization and the level of industrial activity, measurements show the median values of electrical noise in the range 10 to $40 \mathrm{~dB}$ higher than the ITU-R reference for the MF band. Local surveys should therefore be conducted to establish the extent and level of these environmental factors prior to any detailed planning in the MF band.

\section{Conclusions}

A MF radio propagation tool has been developed and subsequently utilized to specify the technical parameters of a single frequency network broadcasting digital radio to the entirety of Greece. Transmitter locations have been carefully selected and simulations have been made for appropriately chosen planning parameters suggested by the ITU. It has been shown, that using as little as $7 \mathrm{~kW}$ total transmitter power, nationwide coverage of sound broadcasting of quality comparable to FM can be achieved.

\section{This paper was presented at Pan-Hellenic Conference on Electronics and Telecommunications - PACET, that took place May 8-9 2015, at Ioannina Greece.}

\section{Acknowledgments}

The authors would like to express their gratitude to Dr. Apostolos Papakonstantinou, Garto GI Lab, Geography Department, Aegean University, Lesvos, Greece, for producing digital versions of the ITU conductivity maps for Greece. The authors wish to acknowledge financial support provided by the Research Committee of the Cental Macedonia Institute of Education and Technology under grant SAT/IC/190516-102/2

\section{References}

1. System Specification ETSI ES 201980 V4.1.1, Jan. 2014.

2. Recommendation ITU-R BS.1514-2 "System for digital sound broadcasting in the broadcasting bands below $30 \mathrm{MHz}$ ", Mar. 2011.

3. Project Mayflower: The DRM Trial, BBC Final Report, April 2009.

4. ITU-R Study Groups, "Results Of DRM Trials In New Delhi: Simulcast Medium Wave, Tropical Band, NVIS And $26 \mathrm{MHz}$ Local Broadcasting", Document 6D/10-E, 28 March 2008.

5. Report ITU-R BS.2144 "Planning parameters and coverage for Digital Radio Mondiale (DRM) broadcasting at frequencies below 30 MHz", May 2009.

6. Recommendation ITU-R BS.1615-1 "Planning parameters for digital sound broadcasting at frequencies below $30 \mathrm{MHz}$ ", May 2011.

7. Recommendation ITU - R P.372-13 "Radio noise", Sep. 2013.

8. Rotheram, S. "Ground-wave propagation", Proc. IRE, 128, Part F, pp. 285-295, Oct. 1981

9. Millington, G. "Ground-wave propagation over a homogeneous smooth earth”, Proc. IEE, 96, Part III, pp. 53-64, 1949.
10. Recommendation ITU - R P.832-3 "World Atlas of Ground Conductivities”, February 2012. 